appreciate a good laugh. She reads, writes, and crochets a little, and she is able to use a knife and fork, although there is some difficulty in accurately gauging the position of the mouth when food is conveyed to it on the fork. Sensation is generally good, and the patient considers that of the right half of the body to be more acute than that of the left. The only abnormality seems to be a deficiency of sensation in the feet and in the lower half of both legs, this being due apparently to the local condition of the skin and to deficient circulation. She cannot walk even with help, and on attempting to do so she drags her legs along the floor. The thighs can be flexed but not the ankles, the foot is extended, and flexion by manipulation of the ankle-joint is deficient. Irregular, jerky movements of the head and neck are present, but none of the legs, and none of the arms except on attempted action to lay hold of anything, when the object is often missed, and the same non-success attends an attempt to touch the tip of the nose with the finger, and the attempted approximation of the corresponding finger-tips of the two hands with the eyes closed, while she has suffi. cient control over her hands to be able to crochet fairly well. Her speech, though thick, laboured, and hesititing, is perfectly intelligible. The tongue is protruded straight, though there is the same jerky irregularity of it as is present in the head and neck. The plantar reflezes are increased, the knee-jerks are absent, and there is no ankle clonus. The rectal sphincters are natural, the bowels requiring an occasional aperient. The bladder shows no loss of control, but there are occasional attacks of retention of urine lasting for two or three days and requiring the use of the catheter, and her mental condition at these times has a tendency towards hysterical manifestations. These attacks of retention have been present for years, sometimes following menstruation, an otherwise natural function. There is no implication of the pupil, but nystagmus of slow character is present, being lateral in direction, brought into play by lateral movements, and not present when the eyes are fixed. Most of the power in the muscles of the lower extremities remains in the extensors of the thigh. The joints move freely except the ankles, flexion of these being deficient from shortening of the calf muscles. Knee-joint flexion and extension are easily performed by manipulation of the limb. The muscles are wasted, their place being apparently supplied intrinsically and extrinsically by a deposit of fat, which is present in good quantity over tbe external surfaces of the buttocks. The feet and the greater part of the legs are congested, thickened, and a little puffy, this condition being apparently induced by the helpleseness of the limbs. The second and third left toes are a little webbed. The fingers share in the deficiency of circulation, but not to nearly so great an extent as the feet.

CASE 2. - The patient is thirty-four years of age and is the sister of the patient in Case 1. The symptoms commenced at the age of ten years with difficulty in walking. The patient is dark-haired, her complexion is muddy and thick, and she is of slighter build and less stout than is her sister. Clinically the pitient resembles her sister in all respects as above detailed, but she differs in that her feet are less blue and cdematous, the condition extending only about tbree inches above the ankle-joint and not reaching nearly to the knee as in Case 1, and also that she has no attacks of retention of urine. Her grasp is strong when she lays hold of anything and she is able to make trimmings for the dead. She was confined on July 27th, 1882, the child, a male, being stillbcrn. Labour was premature (at the eighth month) and was followed by considerable hæmorrhage. At this time she could walk with difficulty. On Dec. $25 \mathrm{th}, 1895$, she fell and fractured the neck of the left femur. It united firmly, but she has not recovered the previous use she had of the limb and it is now practically helpless, whereas the right limb can be flexed at the hip-joint. On examination the leg is an inch and a quarter shorter than the right. Flexion, eversion, and rotation outwards of the hip-joint are all deficient, and when lying on the bed the foot is everted. The great trochanter is a little prominent and somewbat thickened, and its upper extremity is higher than it should be. There is little difference in the circumference of the two limbs, as the right and left calves in greatest circumference measure respectively ten and three-quarter inches and ten and a half inches.

In both cases the heart, lungs, abdomen, and urine are natural, there are no visceral crises, and ocular paralysis is absent.

Herne Bay.

\section{LOCAL HOT DRY-AIR TREATMENT IN ACUTE AND CHRONIC GOUT.}

\section{By W. KNOWSLEY SIBLEY, M.A., M.D., B.C. CAMB.,} M.R.C.P. LoND.,

SENIOR PHYSICIAY TO ODT-PATIENTS AT THE NORTH-WEST LONDON HOSPITAL.

THIS new treatment of joint and other affections by the localised application of very hot dry air I fully described in THE LANCET of Aug. $29 \mathrm{ch}$ of last year. Further experience has confirmed the promising results obtained in the variety of cases then recorded, and the opinion expressed with regard to them. I now wish to draw attention to the action and results of this treatment in a consecutive series of cases of gout, with the belief that the profession will welcome any advance in the present unsatisfactory method of dealing with a disease which directly or indirectly affects such numbers of individuals. The affected limb is left in a tem. perature of up to $300^{\circ} \mathrm{F}$. from half an hour to an hour. During this time, first the limb treated, and then the whole body breaks out into a profuse perspiration, and the pulse, and to a lesser degree the respirations, are increased in frequency. The general effect on the system is considerable, and is evidenced by the fact that the body temperature is raised often one, two, or even more, degrees Fahrenheit, as shown by a thermometer placed in the mouth. In the series of cases of gout which follow no selection of patients has been made; all, therefore, which came for treatment are described in chronological order.

CAse 1. Acute gout - The first case was a man, aged sixty-five years, who came under observation on June 3rd, 1896. The patient had suffered from acute attacks of gout on and off for many years, the attacks often lasting for several months. A year and a half previously he had had a bad attack of pneumonia followed by a prolonged attack of gout in both feet and one knee, which lasted for three months. The present attack had commenced a few days before in the left elbow, which became swollen and the skin tense, red, and very painful; the back of the hand was also swollen, the knuckles being hardly visible and the veins much engorged. After the first treatment by the bot air the parts about the elbow were not so tense, there was less cedema over the back of the hand, and the veins were not so distended. The patient had a return of the atrack in the evening in the elbow, accompanied by severe pain ; after a bath on the following day the elbow appeared less inflamed and could be moved better; the pain was also greatly relieved. On the evening of June 5 th the arm again became more painful, but the patient had a good night; the following day there was some effusion into the tissues below the elbow. Treatment again produced considerable improvement of the parts. By June 8th the gout had quite subsided in the elbow; there was no pain or effusion left, and the joint could be freely moved. During the night the index finger became inflamed; this became more comfortable after treatment and the swelling rapidly subsided. The patient went into the country for a change. In April, 1897, the patient had remained quite well and had been free from attacks of gout since the treatment. During the treatment he was taking alkaline medicine and a saline aperient

CAsE 2. Acute, sub acute, and chronic gout associated with eczema.-This patient was a lady, aged fifty years, who commenced this treatment on June $25 \mathrm{th}, 1896$. There was no history of gout or rheumatism in the family; her father lived to the age of ninety years, and her mother lived seventy-three years and suffered from eczema. Her paternal great.grandfather lived to the age of 105 years. The patient when young had excellent bealth till the first attack of gout, twenty-four years ago, in the right great toe, when she was laid up for a fortnight. From tbis time she had recarrent attacks of acute gout every three or four months. For many years it was confined to the feet. It first appeared in the knees twelve years previously and in the hands for the first time seven years previously. The attacks had been much worse the last six years; she was often laid up in bed for from six to ten weeks, and in acute pain. Five years previously she had an attack of eczema in the feet, and this bad troubled her on and off ever since, never having disappeared. Eight years ago the chalky gouty deposit in the right great 
toe broke down and discharged for twelve months, and this place had reopened on and off several times since. In October, 1895, the deposit in the left index finger discharged for four months, and about the same time the left great toe also discharged. From October, 1895, to June 25th, 1896, the patient had been laid up in bed, being unable to move because of pain, although taking large quantities of medicine. The left index finger was mach deformed, and chalky deposit was seen beneath the thinned skin. The little finger of the right hand was also much deformed; there was chalk stone deposit in the middle finger of the left hand. The left knee was extremely painful and tender, movement was very limited on account of pain, and there were some deposits to be seen and felt on the anterior surface of the patella. B th the feet were swollen and covered with an irritable form of eczema, which, especially on the left, extended some way up the leg; both great toes were much deformed, tumid, and inflamed, very red and acutely painful; they were both dislocated outwards and the lelt was discharging thick pus and chalky matter and looked very angry. There was considerable puffiness about the ankles and pain on the slightest aitempt at movement. There were deposits in both ears. On June 25 th the left leg was placed in the cylinder. The pain was soon relieved by the heat, and when taken out she was able to move the limb freely and could even walk on it without pain; the eczematous condition was also better. The next day she reported that she had slept all night, that the knee was almost well and the foot much less painful, and that there had been no irritation from the eczema, although for the first time for four months she had not used any ointment. She had a second bath and was again much relieved. She returned for a third bath on July 6th, when she reported that she had bad ano her attack of eczema which was very distressing. After treatment the intense irritation was much relieved; the toe continued to discharge freely. The patient's foot was too bad to allow her to come for a bath from July 8th to 23ad, when, after a bath, the part was much better, and she felt very comfortable. Bg Aug. 20 th the patient had had ten baths. The fourth finger was now decidedly smaller, and the chalky deposit in the knuckle of the second finger was dispersing. On the 25th the patient had her eleventh bath, and said she was able to play the piano for the first time for six months. On the $26 \mathrm{th}$, after the twelfth operation, the following note was made: "The various chalky deposits are decreasing quickly and becoming much softer; the fingers are more flexible and the whole hands are of a much better shape." On Sept. 2 nd the patient informed me that she had been trying her voice and had found that it was quite restored; she had not sung for eighteen months. She was also able to run up and down stairs; the hands continued to improve. On the $29 \mathrm{sh}$ the patient had her twentieth bath. She had had a bad cold, and on the previous day, after doing some needlework, she felt a sudden sharp pain from the right shoulder to the tip of the second finger on the right hand, and this had become stiff and painful. Now the elbow was also painful, the tissues about it being tense and swollen, and tender to the touch. All these symptoms were much relieved by another application of the hot air. The next day she reported having had a good night, and there had been no pain in the finger or elbow since; she had been able to dress herself. On Dec. 16th the patient again presented herself, not having attended since September owing to numerous domestic worries. She had been well with the exception of an attack of pain under the knees and down the calves (? phlebitis) in November. At this time the patient was fairly well in herself, but had some gout about the ankles and heels; the eczema had quite disappeared from the feet. The right elbow was entarged, red, and painful. On the 27th the swelling about the left elbow, which was present last time, had increased and had become extremely painful. On the 29 th the patient reported that her left elbow was much less painful for two days after the last bath, but it had then again become worse ; the right elbow had not since been painful. On the 30 th the left elbow was very red, swollen, and painful over the olecranon process; there was also a good deal of pain up the arm (lymphatic) and also in this axilla. The pain and congestion of the whole was much relieved by the bath. The next day the patient reported that the elbow again became painful during the evening, but she had had a good night and was feeling much better. During the next few hours the left elbow kept very painful and the skin broke on Jan. 5th, 1897, and it then discharged slightly. A day or two afterwards the right arm became painful, then the left became still more inflamed, and the patient had to keep in bed. She had had no sleep for several nights, so she was taken in for treatment on Jan. 13th. The left arm, which was greatly inflamed and red from the elbow to the middle of the arm, was placed in the cylinder, after which the patient fell asleep. Having slept for three hours, she awoke feeling much more comfortable. She did not have a good night, the elbow discharged very freely a thick chalky material, but in the morning the swelling and inflammation had considerably subsided. The whole condition rapidly improved during the next few days. By Jan. 21st the patient was well and she returned home. The elbow had ceased to discharge and the movements of the joints were unimpaired. She stated that the last time ber finger discharged it continued to do so for six months. In April, 1897, the patient had continued quite free from gout since January, and there had been no return of the eczema; her general health had also greatly improved. CASE 3. Sub-acute gout, chronic bronclitis, and albuminuria.-This patient, who was first s€en on Sept. 2lst, 1896, had probably inherited gout from his mother's side of the family. This patient, a painter by trade, had had severe colic fourteen years ago, and was sixty-one years of age. The first attack of gout occurred ten years ago in the right foot, when he was laid up for a fortnight; a second attack came on three years later, and for the last three years he had had repeated attacks, especially in the ankles. When he came to the hospital the right foot $\mathrm{w}$ is swollen, very tender, and painfal, the skin being red and much inflamed; both legs were cedematous. Internal remedies were presciibed. On Oct. 12th the general condition was much the same. The cough and bronchitis continued troublesome, with a good deal of expectoration in the early morning. There were general bronchial rhonchi. especially at the right apex. The urine was clear, of specific gravity 1012, and contained albumin. The second heart sound was accentuated. The condition of the foot was much about the same, the patient being hardly able to get about, and then only with great pain. On the 13th the first hot-air application was given, no change being made in the medicines. The foot was much easier and he could move it more fully after treatment. By the 19 th he had had three baths. The foot was much better, and the bronchitis was also much improved; there appeared to be less albumin than formerly. He had slept much better since the baths were administered. On the 20th the patient stated that his foot felt quite well; he had no pain whatever. He took a mixture of gentian and bicarbonate of soda all through the treatment.

CASE 4. Acute gout.-This case of acute gout in a man, affecting the left ankle, was greatly relieved by three baths.

CASE 5. Acute gout -A man, aged forty-three years, was first seen on Nov. 13th, 1896. His father died trom rheu. matism and Bright's disease. Gout occurred for the first time ten years ago, and the attacks had been repeated at least once a year ever since. This attack came on two days previously with great pain, chiefly in the left foot. He was given an alkaline mixture of soda and gentian and the hotair baths. The patient felt great relief from the first bathhe could bend the foot with comfort afterwards and the pain was much less. On the 15 th the foot had been much better. There was no pain but some stiffness about the ankle when walking. After another bath the foot felt quite well and the stiffness had disappeared and did not return. On April 3rd, 1897 , the patient wrote to say that he had not had a return of gout in the feet and had continued at work ever since the previous November.

CASE 6. Acute gout.-A man, twenty-four years of age, came under observation on Dec. 7th, 1896. There was no family history of gout or rheumatism. Fourteen weeks previously the patient had suffered from inflammation of the eyes, which lasted three weeks, followed by pain in the back, which kept him in bed for three weeks; afterwards the pain settled in the knees, the left big toe, and then in the left hand and arm. Between Dec. 7th and 21 st he had four baths, and in addition a mixture containing bicarbonate of soda and iodide of potassium. By Jan. 4th, 1897, he was quite free from gout.

CASE 7. Gont, bronchitis, heart disease, albuminuria.This patient, who was a woman, aged sixty-five years, came under observation on Nov. 23rd, 1896. Her maternal grandmother, who suffered from gout, died when over eighty, and her mother died at ninety-three years of age. She was quite 
well till forty-three years of age, when she had the first attack of gout in the left great toe. She had had frequent attacks since in the feet, ankles, knees, elbows, and hands. For the last eighteen years she had also suffered much from bronchitis. On Dec. 8th she was admitted into the hospital. At this time the patient was very weak and suffered much from dyspnœa; the pulse was rapid (120) and small; the cardiac dulness was increased; there was a soft systolic murmur at the apex and a much louder one at the base, which murmur was readily heard in the vessels of the neck; the heart sounds were very feeble; there were general bronchial rhonchi over both the lungs. The urine was of specific gravity 1010, acid, and clear, and there was some albumin present. The feet were much distorted from old standing gouty changes; there was a large nodule in the left metatarsal phalangeal articulation of the big toe. There was very little movement in the knee-joints, and there was thickening about the heads of the tibiæ. The joints of all the fingers were enlarged, and the fingers defiected to the ulnar side. There was a large firm nodule over the right olecranon and hard nodules on the outer side of the olecranon of the left elbow. The patient had had little or no sleep for weeks, and the breathing was rapid and shallow. On Dec. 11th, after the first bath, the patient seemed to be a little easier. By the 14th the breathing was better after the bath, but she had not slept much. By the 21st the right hand was not so painful and the cough was a little easier the patient still felt very weak $\mathrm{z}_{\mathrm{z}}$ and her skin did not act well. She gradually improved, and left the hospital on Jan. 7th, 1897 .

CASE 8. Sub-acute gout. - The patient, who was a cabdriver, thirty-eight years of age, was first seen on Jan. 4th, 1897. He was treated in the same way as the patient in Case 6, being given two baths, and by Jan. 14th he was free from pain.

CASE 9. Acute gout.-The patient, a woman aged sixtytwo years, came to the hospital on Feb. 22nd, 1897, and gave no family history of gout or rheumatism. She had congenital absence of the first, second, and third fingers of the left hand. Eighteen years ago she had her first attack of gout in the right great toe, and she had had repeated attacks, often twice a year, ever since. The present attack came on a week ago in the left shoulder, then the right leg, and three days later the right hand became involved. The whole hand was much swollen, very red, and the skin much inflamed and acutely painful ; there was some inflammation of the left ankle. She also suffered from chronic bronchitis. The first hot-air application was administered on the 23rd, with the result that the hand was less swollen and felt much easier after the bath. A second bath was given on the 25th. The fingers continued to be very stiff, but there had been much less pain in them. A third application was given on March 1st, when the patient said that she had slept much better and the pain had been much relieved. After this she considered herself cured and returned to her occupation on March $10 \mathrm{th}$. On admission to hospital she was ordered 5 grains of iodide of potassium three times a day and ten drops of colchicum vine every night, and on March Ist she had an alkaline mixture containing five grains of iodide of potassium three times a day.

CASE 10. Acute gout.--This patient was a salesman, who came to the hospital on March 21st, 1897, suffering from acute gout in both hands, which was quite relieved by three baths and an alkaline mixture.

It is important to note that nearly all of the above cases were treated as hospital out-patients, and that therefore it was impossible to regulate or in any way control their daily habits as to diet, \&s. Most of the patients were also only treated during an attack of gout; it was difficult to persuade them to undergo treatment by the hot air between the attacks, even with a fair expectation of keeping off a return of the disease. For instance, in Case 2, although many promises were made, something always interfered to prevent the continuance of regular treatment. In spite of the considerable shortening of the attacks and of the improvement in her general condition, freely admitted by the patient, only the return of pain would persuade her of the need to continue with the hot-air applications. My experience with these cases is that directly the pain subsides the patient returns to work, and neglects to take medicine or the ordinary diet precautions. The cases above described contain examples of gout in its various stages, from the most acute, through the sub-acute, to the very chronic, these latter, sometimes with acute exacerbations, occurring from time to time. Taking first the very acute attacks (Cases 1, 4, 5, 6, and 9), in all of these the local intense pain and congestion were almost imme diately relieved by the hot air; very obvious difference in the appearance of the part was seen after the application and the relief from the intense agony of an acutely inflamed gouty joint was most marked. But gout, like rheumatism, is always prone to reappear; and it was found in Cases 1 and 6, that the acute inflammation in the part first affected rapidly improved under the local treatment, and in the course of a few days another deposit, but one not necessarily so severe, appeared in another part, sometimes the corresponding limb of the opposite side. Treatment would again rapidly disperse this second deposit, and in no instance did it afterwards follow in another joint. It would seem that in nearly all the cases the attack under hot-air treatment was very much shorter than the previous attacks which had been treated only by internal remedies (Cases 1, 2, 4, and 5). Often, in fact, the attack which previously had lasted for weeks under this method lasted only for the same number of days. In some (Cases 2, 6, 8, and 9) internal medicines, such as small doses of colchicum and iodide of potassium, were also given ; in others only a simple alkaline mixture (with possibly a slight aperient such as Condal water) was administered, as in Cases $1,3,5$, and 10 . Nevertheless, in all of these cases the relief from pain and the rapid and complete disappearance of the gout were most marked. Case 2 very well illustrates the treatment of very chronic gout, with gouty or chalk stone deposits; the deposit in one finger, for instance, being so bad that at one time the medical attendant had wished to amputate the digit, and yet after several applications of heat the deposit gradually disappeared, and so also the deposits about the heads of the larger bones became distinctly lessened.

A few remarks are necessary with regard to the mode of action of this treatment. The heat applied locally to the inflamed gouty part causes an increased circulation in the area, bringing a larger quantity of blood to the seat of the lesion and at the same time taking away a larger quantity of blood probably more or less saturated with the bodies causing the local deposits. These deleterious products being in the general circulation, so to speak, dissolved out of the seat of the inflammation should in the normal course of events largely find their way out of the body through the kidneys, hence all should be done during the course of treatment to assist these organs to act as freely as possible. Some interesting observations on this part of the subject have recently been made in the clinics of Professor Landouzy at the Laënnec Hospital, and Dr. Déjerine at the Salpêtrière Hospital, in Paris, who are experimenting with the Tallerman treatment, the results of which were published by Dr. Chrétien. ${ }^{1}$ They found, for instance, that in one case of ordinary gout the daily elimination of uric acid, which after the fourth bath was 57 centigrammes, rose after the ninth bath to 89 centigrammes; and in another case of arthritis the daily co-efficient of urea had changed from 20 grammes 97 centigrammes before treatment to 25 grammes 50 centigrammes after the treatment had been administered. They also found an increased excretion of all the salts, especially the chlorides. This increased excretion through the kidneys is probably the explanation of the great benefit of this hot-air treatment in cases of gout. The results in the French hospitals were obtained entirely by this external method and without the exhibition of drugs of any kind. I feel no doubt of the great benefit this treatment will show in cases of what are described as " constitutional goutiness," without necessarily any local objective lesion. Upper Brook-street, W.

1 La Presse Médicale, Dec. 26th, 1896.

Pauperism in the West of England.-In the first half of 1896 a sum of $£ 206,609$ was spent upon in-door and out-door relief in the five south-western counties. This is an increase of $£ 1250$ for the corresponding period of 1895 . Devonshire is the only county which shows a decreased expenditure, the decrease amounting to £1224. Devonshire spent $£ 14,378$ on in-door and $£ 58,426$ on out-door relief. Cornwall expended $£ 5689$ on in-door and $£ 23,502$ on out-door relief. Somerset's total represents an increase of 2.9 per cent. over the beginning of 1895 , the sum in 1896 standing at $£ 12,807$ for in-door and $£ 41,931$ for out-door relief. 


\section{A thitror}

\author{
or
}

\section{HOSPITAL PRACTICE, BRITISH AND FOREIGN.}

Nulla autem est alia pro certo noscendi via, nisi quamplurimas et morborum et dissectionum historias, tum aliorum tum proprias collectas habere, et inter se comparare.-MoRGAGNI De Sed. et Caus. Morb., lib. iv. Procmium.

\section{ST. BARTHOLOMEW'S HOSPITAL.}

A CASE OF PY \&MIA AND ACUTE SUPPURATIVE ARTHRITIS WITH COMPLETE RECOVERY OF THE JOINTS.

\section{(Under the care of Mr. Buturn and Mr. Lockwood.)}

OF the many varieties of streptococcus infection our present knowledge seems to suggest that the serum treatment is of more value in the local forms, such as erysipelas, ${ }^{1}$ than in those cases such as pyæmia, in which the infection is probably more general. In the following case, however, the earlier injections were distinctly followed by marked improvement. The result of the active treatment of the joints affected is extremely satisfactory, and contrasts greatly with the treatment of pyæmic joints a few years ago, when ankylosis was generaily the best result that could be looked for after the infection of a joint with pyæmia. For the notes of the case we are indebted to Mr. J. Preston Maxwell, house surgeon.

A married woman, aged thirty-three years, was admitted to St. Bartholomew's Hospital on Feb. 13th, 1897, at the end of an attack of acute suppurative tonsillitis, which, she said, began three weeks previously. Almost from the beginning of her illness she had had various complications. The left knee-joint was acutely inflamed and full of fluid, but the skin over it was normal, and the slightest movement caused her intense pain. All the other joints were healthy. There was slight dulness with some pleural friction at the right base, indicating a pleurisy of the right side, which was said to have commenced soon after the tonsillitis, and was now subsiding. The heart was particularly examined and no murmur or dilatation could be discovered. The urine was acid, of normal specific gravity, and without any trace of albumin. Her temperature was $101.5^{\circ} \mathrm{F}$., her pulse was 132 , and her respirations were 34 . It was clear that the disease in the knee-joint was both acute and progressive and therefore it was decided to ascertain the nature of the fluid contained in it. In the evening of the day of admission an anæsthetic was given, and, the skin having been disinfected, the needle of a sterilised exploring syringe was introduced. Pus was withdrawn; it was thin and quite odourless (two peptone broth tubes and an agar-agar tube were inoculated with the pus, but nothing grew). An incision on each side was made into the knee-joint and the remainder of the pus washed out; the joint was syringed first with a solution of biniodide of mercury ( 1 in 500 ), and then with a weaker solution of the same antiseptic ( 1 in 4000). The synovial membrane was seen to be inflamed and the articular cartilages felt velvety. A rubber drainage tube was inserted on each side of the joint, a large dressing of iodoform, iodoform gauze, and sal alembroth wool was applied, and the limb was placed on a straight back splint. On the following day (Feb. 14th) the patient's condition was about the same, though her temperature was slightly lower $\left(101^{\circ}\right)$; the joint was still very painful. This was attributed to the continuance of septic inflammation and to the fact that the splint had to be removed for dressing and irrigation with biniodide of mercury solution ( 1 in 4000), which was done twice daily. Therefore on Feb. 15th an anæsthetic was again given and the knee-joint thoroughly irrigated with the weaker biniodide solution, a determined effort being made to disinfect the cavity of the joint. The limb was then swung on a straigh back splint with a foot-piece, the splint being covered with a mackintosh to allow of daily irrigation without disturbing the limb. Before the anæsthetic was given it was noticed that there was a well-marked systolic murmur at the heart's apex. On the 16 th the patient was worse. She was not sleeping well and her temperature had risen to $104.6^{\circ}$,

1 Marmorek: Annales de l'Institut Pasteur, Nov. 7, 1895, and Tison: Journal de Médecine de Paris, April 26th, 1896. the pulse was 140, and the respirations were 32. The pleural friction could no longer be heard at the right base, but it was very distinct at the left apex in front. In addition to the systolic murmur there was a distinct diastolic bruit to be heard at the heart's apex. She was taking fluid food fairly well and the bowels were acting naturally. On the 17th it was clear that her condition was becoming highly critical. Hitherto the absence of rigors had been a peculiar feature of the case, and even now she did not have a distinct shivering fit; she had felt alternately hot and cold during the night, and her temperature, which had rapidly fallen to normal, was again rising fast. Her tongue was dry, and since the early morning she had been retching and vomiting. Slight subsultus tendinum was observed, and purpuric spots were noticed over both hands and forearms and the right leg. Her mind was clear, and delirium was absent. Although there was no clear evidence that it was a case of streptococcus poisoning, yet the probability seemed so strong that it was decided to commence the administration of antistreptococcic serum. At 2.45 P.M. the first injection of the serum was given into the substance of the rectus abdominis, with marked effect. The temperature fell half a degree, to $99 \cdot 6$, the vomiting and retching ceased, the colour and aspect improved, the subsultus tendinum disappeared, and the tongue became moist. The respirations came down from 34 to 28 per minute, and the patient volunteered the statement that she "felt better." The knee looked well. (At this time blood cultivations were taken from the finger, but no growth was obtained.) At 8 P.M. another injection of 10 c.c. of the antistreptococcic serum was given. She was keeping better, but during the night the temperature rose steadily, and the injection seemed to have had but little effect. She did not sleep well. At 3 A.M. on the 18th a third injection of 10 c.c. of the serum was given into the rectus. At the time she was delirious, and the injection did not prevent the temperature rising steadily. At 8 A M. a fourth injection of a similar quantity of serum was given with no apparent result, and at 4 P.M. a fifth injection of the same amount was administered. The patient was now delirious, passing everything under her, refusing medicine, but taking liquid food fairly well. The temperature was $102^{\circ}$, the pulse was 140 , and the respirations were 28. The knee was still irrigated daily, and looked well. On the 19th she was still going down hill; the delirium continued and fresh purpuric spots had appeared on the right leg., Her face was sallow, and her eyes were sunken. The systolic murmur was still audible at the apex. At 11.30 A.M. 20 c.c. of antistreptococcic serum were injected into the rectus, but as this injection had no effect whatever on her condition, injections were now discontinued. She was ordered 5 grains of sulphate of quinine in half an ounce of brandy, to be given every four hours. She continued to grow worse during the day, and at 4 P.M. sudden paresis of the right side of the face appeared, with slight twitching of the muscles of the face and neck. She was semi-comatose. The knee, which was still irrigated daily, looked well. On the 20th slight improvement had set it, she was rational, the paresis and twitching of the face had quite disappeared, and there were no fresh purpuric spots. She had taken the quinine well and had no signs of cinchonism. The tem perature was $103^{\circ}$. The improvement was maintained, and she slept fairly at night, and her temperature, pulse, and respiration hardly changed for the next tbree days. On the 23rd the right knee became fall of fluid. It was explored, opened, and drained in the same way as the left. The pus was of the same kind and yielded no growth on cultivation. A splint like that used for the left leg was used for this knee also. Both knee-joints were irrigated daily. From this time the patient steadily improved. The temperature came slowly down to the normal, which it reached on March 12th. The quinine was discontinued on Feb. 26th. The drainage-tubes were removed from both knees between March 1st and 5th and the wounds were allowed to close. As soon as they were healed the splints were removed and she was encouraged to use and bend the knees as much as possible. Massage was also employed. After the falling of the temperature she began to put on flesh and rapidly improve in her general health. By April 15th she could walk round the ward unaided, except by a stick, and could bend her knees through an angle of $70^{\circ}$; all the purpuric spots had quite disappeared, and no heart murmur could be heard. She was then discharged from the hospital; about the middle of May she was 